\title{
A Pseudo-Boolean Optimization for Multiple Criteria Decision Making in Complex Systems
}

\author{
Bahram Alidaee $^{1}$, Haibo Wang ${ }^{2, \star}$, and Yaquan $\mathrm{Xu}^{3}$ \\ ${ }^{1}$ University of Mississippi, University, MS 38677, USA \\ balidaee@bus.olemiss.edu \\ 2 Texas A\& M International University, Laredo, TX 78041, USA \\ hwang@tamiu.edu \\ ${ }^{3}$ Viginia State University, Petersburg, VA 23806, USA \\ yxu@vsu.edu
}

\begin{abstract}
In complex system problems, a Decision Maker (DM) is often faced with choosing a subset of alternatives from a bigger set. This process is known as multiple criteria decision making (MCDM). Examples of MCDM include decision making in human resource management, water resource management, environmental management and site selection, energy policy issues, portfolio selection, transportation and routing selection, student admission. In general, there are several criteria that need to be satisfied; however, they are usually at least partly conflicting. In this study, we propose a pseudo-boolean approach for multiple criteria decision making on complex system problems. The computational results illustrate both robustness and attractiveness of this solution approach.
\end{abstract}

Keywords: Pseudo-Boolean Optimization, Multiple Criteria Decision Making.

\section{Introduction}

Complex system problems are often hard to model and some of them can be represented by nonlinear objective functions with interconnectedness among multiple dimensional spaces. Conventional mathematical programming approaches are sometimes unable to solve it directly. It is conventional wisdom to apply the combination of artificial intelligent, evolutionary computation, system dynamics and heuristic such as genetic algorithm and ant colony to solve these problems [1 2 , 3 4,5,6. Other approaches using chaos theory and morphological analysis are reported in the literature [7/8. In this study, we apply multiple criteria decision making model to complex systems which can be solved by a pseudo-boolean optimization approach.

pseudo-boolean formulations are widely known for their ability to represent a rich variety of important discrete problems, which are often NP-hard. Hammer and Rudeanu [9] introduced pseudo-boolean optimization in their early work and presented a dynamic programming procedure for solving certain problems.

\footnotetext{
^ Corresponding author.
} 
In their paper, they gave a definition of a first-order derivative and indicated its use in solving discrete pseudo-boolean optimization problems. Detail surveys of pseudo-boolean optimization are given by Boris and Hammer 10 and Crama and Hammer [1].

In this paper we extend this notion of first order derivatives by defining higherorder derivatives for discrete pseudo-boolean optimization. Moreover, in the context of changing $r$ elements of $x$ at a time (the so-called $r$-flip moves for $r=$ 2 and 3 ), we present closed-form formulas that allow 'efficient' implementation of such compound moves. Then, for the important special cases of quadratic and cubic optimization, we define a general $r$-flip move that allows efficient implementation of multi-exchange neighborhood search process for solving such problems. Finally, we illustrate the use of such moves by applying variants of simple search processes based on $r$-flip moves (with $r=1$ and 2) to a test bed of complex system problems. The paper then concludes with summary and a look ahead to future research.

\section{Multiple Criteria Decision Making}

In complex system problems, a Decision Maker (DM) is often faced with choosing a subset of alternatives from a bigger set. This process is known as multiple criteria decision making (MCDM). Examples include decision making in human resource management, water resource management, environmental management and site selection, energy policy issues, portfolio selection, transportation and routing selection, student admission. In complex system, there are several criteria that need to be satisfied; however, they are usually at least partly conflicting. If an alternative is selected in conjunction with other alternatives it has different (positive or negative) effects on a specific criterion. Thus, selected alternatives have interaction effects on different criteria. Often a DM in practices uses a simple weighted linear function to rank alternatives then select top few. Not considering interdependencies among alternatives can lead to an undesirable outcome. For an illustration consider the following example on Waste Disposal Location (WDL) problem taken from Rajabi et al. [12. In WDL problem, the objective is to identify the two best among five potential sites of equal capacity. Three criteria for success has been identified, (1) proximity to population, (2) infrastructure requirements, and (3) environmental risk. The WDL problem is characterized by certain interdependencies among alternatives. Building a new road near sites 4 and 5 could serve both sites; if both sites are selected, a saving in infrastructure investment will be obtained. If both sites, 4 and 5, are selected there is an increased of $10 \%$ on the infrastructure investment criterion. Similarly, if sites 1 and 2 are simultaneously selected then a single power plant facility may be built for both, taking advantage of economy of scale. In that case, a positive energy of $30 \%$ is estimated when it comes to infrastructure investment criterion. Finally, when it comes to environmental risk criterion, selection of site 4 and 5 together has a negative effect of $30 \%$. The scenario is illustrated in the Figure 1, and data is presented in Table 1. 

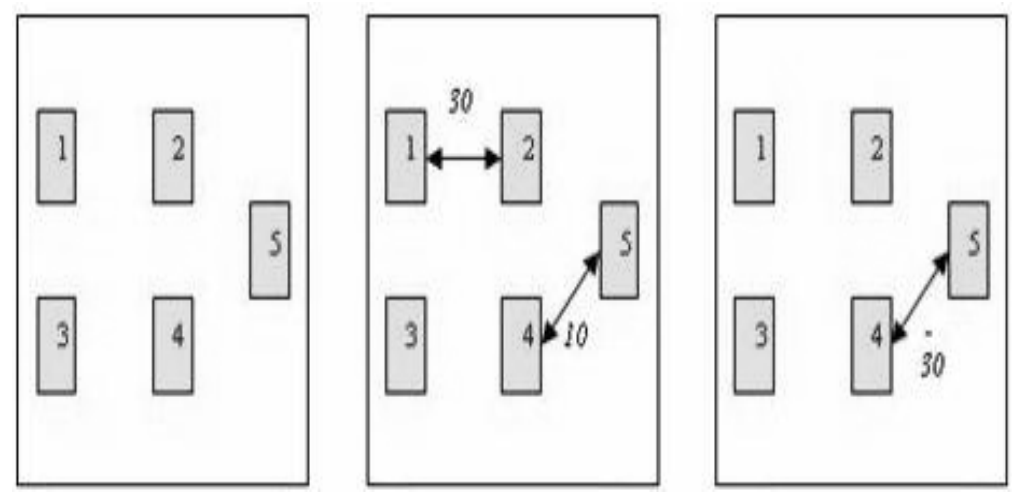

Fig. 1. WDL example: Interdependence actions and synergy levels. (Left: Proximity to population, Middle: Infrastructure requirements, Right: Environmental risk).

Table 1. Normalized consequences of five feasible sites

\begin{tabular}{|c|c|c|c|c|c|c|c|}
\hline \multirow{2}{*}{ Weights } & Criteria & \multicolumn{5}{|c|}{ Alternatives } \\
\cline { 4 - 8 } & & & $a^{1}$ & $a^{2}$ & $a^{3}$ & $a^{4}$ & $a^{5}$ \\
\hline 1 & 0.23 & Population & 0.45 & 0.45 & 1.00 & 0.55 & 0.84 \\
\hline 2 & 0.39 & Infrastructure & 0.80 & 0.70 & 0.75 & 0.83 & 0.83 \\
\hline 3 & 0.38 & Environmental Risk & 0.60 & 0.87 & 0.50 & 0.75 & 0.60 \\
\hline \multicolumn{2}{|c|}{ Additive Value } & 0.644 & 0.707 & 0.713 & 0.735 & 0.745 \\
\hline
\end{tabular}

The following notations are adopted in the paper:

$N=\left\{a^{1}, \ldots, a^{n}\right\}$ is set of alternatives.

$a^{i},(i=1, \ldots, n)$ is alternative $i$.

$P=\{1, \ldots, n\}$ is set of criteria.

$c_{k}^{i}$ is effect of alternative $i$ on criterion $k$.

$S_{k}$ is a set of alternatives that if selected together have some positive or negative effect on criterion $k$.

$\gamma\left(S_{k}\right)$ is the amount of effect (positive or negative) of an interacting set $S_{k}$ on criterion $k$.

$w_{k}$ is weight associated with criterion $k$.

$v(S)$ is total payoff of a subset of alternatives $S \subseteq N$.

$x_{i}$ is equal to 1 if an alternative $a^{i}$ is selected and 0 otherwise.

To illustrate the notations, consider Table 1 , we have 5 alternatives $N=$ $\{1,2,3,4,5\}$, and 3 criteria $P=\{1,2,3\}$. Alternative $A^{2}$ has effects of $c_{1}^{2}=$ $0.45, c_{2}^{2}=0.70$, and $c_{3}^{2}=0.87$ on criterion, 1,2 , and 3 , respectively. Weights of criteria are $w=\left(w_{1}, w_{2}, w_{3}\right)=(0.23,0.39,0.38)$. Considering criterion 2 there are two interacting alternatives, $S_{2}=\left\{a^{1}, a^{2}\right\}$ and $S_{2}=\left\{a^{4}, a^{5}\right\}$, with interaction effects (positive energy) of $\gamma\left(\left\{a^{1}, a^{2}\right\}\right)=0.30$ and $\gamma\left(\left\{a^{4}, a^{5}\right\}\right)=0.10$. If no 
interdependencies are considered among the alternatives then the payoff value is defined as a simple additive function, for example, for $S=\left\{a^{i}, a^{j}\right\}$ we have

$$
v\left(a^{i}, a^{j}\right)=v\left(a^{i}\right)+v\left(a^{j}\right)=\sum_{k=1}^{3} w_{k} c_{k}^{i}+\sum_{k=1}^{3} w_{k} c_{k}^{j} .
$$

In this case, considering all possible set of 2 alternatives and selecting a subset with the largest payoff we have optimal solution as $v\left(a^{4}, a^{5}\right)=v\left(a^{4}\right)+v\left(a^{5}\right)=$ $0.735+0.745=1.48$. However, this is a simplistic view of the situation. If we consider interdependencies between alternatives and taking into accounts positive and negative energies we have a dynamic weight for each criterion when applied to an alternative. The dynamic weight of an alternative depends on its interaction with other alternatives in the selected subset.

\section{$3 \quad$ Model and Solution Methodology}

In the following, we will present a pseudo-boolean formulation of the problem that can overcome both of these deficiencies if appropriate solution procedure is available. The pseudo-boolean optimization for the problem can be presented as a maximization problem given below [10]

$$
\begin{array}{ll}
\text { Max } & \sum_{i \in N} x_{i}\left[\sum_{k \in P} w_{k} c_{k}^{i}\right]+\sum_{k \in P} \sum_{s_{k} \subseteq N}\left(w_{k}\left[\gamma\left(S_{k}\right)\right]\left[\sum_{i \in S_{k}} c_{k}^{i}\right]\right) \prod_{i \in s_{k}} x_{i} \\
\text { s.t. } & \sum_{i \in N} x_{i}=m, \\
& x_{i} \in\{0,1\} .
\end{array}
$$

In this problem, if we want to find any subset of alternatives with largest payoff, then we remove the equality constraint and solve the problem.

To illustrate the optimization problem we use the WDL example presented below.

$\operatorname{Max} \quad .644 x_{1}+.707 x_{2}+.713 x_{3}+.735 x_{4}+.745 x_{5}+.39(.30)[.80+.70] x_{1} x_{2}+$ $.39(.10)[.83+.83] x_{4} x_{5}-.39(.30)[.75+.60] x_{4} x_{5}$

s.t. $\quad x_{1}+x_{2}+x_{3}+x_{4}+x_{5}=2$, $x_{i} \in\{0,1\}$.

Simplifying the above formulation we get

$$
\begin{array}{ll}
\operatorname{Max} & .644 x_{1}+.707 x_{2}+.713 x_{3}+.735 x_{4}+.745 x_{5}+.1755 x_{1} x_{2}-.8916 x_{4} x_{5} \\
\text { s.t. } & x_{1}+x_{2}+x_{3}+x_{4}+x_{5}=2, \\
& x_{i} \in\{0,1\} .
\end{array}
$$

Glover 13 used an efficient 1-flip search implementation and tested it in the context of Tabu Search and Scatter Search.

Definition 1. Let $x=\left(x_{1}, \ldots, x_{i}, \ldots, x_{n}\right)$ be a solution to pseudo-boolean and let $x=\left(x_{1}, \ldots, \bar{x}_{i}, \ldots, x_{n}\right)$ be a solution obtained from $x$ by complementing $x_{i}$. 
Since $Q$ is upper triangular matrix then $(i j)$ th element, $q_{i j}$, is equal to the second derivative of $f(x)$, i.e., $\delta_{i j}(x)=q_{i j}$, with respect to $x_{i}$ and $x_{j}$.

Theorem 1 (Glover et al.[14]). For $x$ and $x^{\prime}$ as given in Definition 1, the change in the value of the objective function is

$$
f\left(x^{\prime}\right)-f(x)=\left(x_{i}^{\prime}-x_{i}\right) \Delta_{i}(x)
$$

Moreover, in any local optimal solution of the pseudo-boolean with respect to 1-flip search we have

either $\left(x_{i}=0 \Longleftrightarrow \Delta_{i}(x) \geqslant 0\right)$ or $\left(x_{i}=1 \Longleftrightarrow \Delta_{i}(x) \leqslant 0\right), \forall i=1, \ldots, n$.

Furthermore, after changing $x_{i}$ to $\bar{x}_{i}$ the update for all $\Delta_{i}(x)$ can be calculated as follows

$$
\begin{array}{ll}
\forall j<i, & \Delta_{j}(x)=\Delta_{j}(x)+q_{j i}\left(x_{i}^{\prime}-x_{i}\right), \\
\forall j>i, & \Delta_{j}(x)=\Delta_{j}(x)+q_{i j}\left(x_{i}^{\prime}-x_{i}\right), \\
j=i, & \Delta_{j}(x)=\Delta_{i}(x) .
\end{array}
$$

In Theorem 2, we extend the above result to general case for $r$-flip search for pseudo-boolean.

Theorem 2. Let $x$ be a given solution of pseudo-boolean and $x^{\prime}$ obtained from $x$ by an $r$-flip move where $S \subseteq V$, and $|S|=r$. Now, the change in the value of the objective function is

$$
f\left(x^{\prime}\right)-f(x)=\sum_{i \in S}\left(\bar{x}_{i}-x_{x}\right) \Delta_{i}(x)+\sum_{\forall i, j \in S}\left(\bar{x}_{i}-x_{i}\right)\left(\bar{x}_{j}-x_{j}\right) \Delta_{i j}(x) .
$$

Furthermore, after changing $x$ to $x^{\prime}$ the update for all $\Delta_{i}(x)$ can be calculated as follows,

$$
\begin{array}{ll}
\forall j \in V \backslash S & \Delta_{j}(x)=\Delta_{j}(x)+\sum_{i \in S}\left(\bar{x}_{i}-x_{i}\right) \Delta_{i j}(x), \text { and } \\
\forall j \in S & \Delta_{j}(x)=\Delta_{j}(x)+\sum_{i \in S \backslash\{j\}}\left(\bar{x}_{i}-x_{i}\right) \Delta_{i j}(x) .
\end{array}
$$

Proof. For two vectors $x^{\prime}$ and $x$ where component $x_{i}$ for all $i \in S$ are complemented we have $f(x)$ and $f\left(x^{\prime}\right)$ as follows:

$$
\begin{aligned}
& f(x)=\sum_{i \in S} x_{i}\left[q_{i j}+\sum_{j \notin S} x_{j} q_{i j}\right]+\sum_{i j \in S} x_{i} x_{j} q_{i j}+\ldots \\
& f(x)=\sum_{i \in S} x_{i}\left[\Delta_{i}(x)-\sum_{j \notin S \backslash\{i\}} x_{j} q_{i j}\right]+\sum_{i j \in S} x_{i} x_{j} q_{i j}+\ldots \\
& f\left(x^{\prime}\right)=\sum_{i \in S} \bar{x}_{i}\left[q_{i j}+\sum_{j \notin S} x_{j} q_{i j}\right]+\sum_{i j \in S} \bar{x}_{i} \bar{x}_{j} q_{i j}+\ldots \\
& f\left(x^{\prime}\right)=\sum_{i \in S} \bar{x}_{i}\left[\Delta_{i}(x)-\sum_{j \notin S \backslash\{i\}} x_{j} q_{i j}\right]+\sum_{i j \in S} \bar{x}_{i} \bar{x}_{j} q_{i j}+\ldots \\
& f\left(x^{\prime}\right)-f(x)=\sum_{i \in S}\left(\bar{x}_{i}-x_{i}\right)\left[\Delta_{i}(x)-\sum_{j \notin S \backslash\{i\}} x_{j} q_{i j}\right]+\sum_{i j \in S}\left(\bar{x}_{i} \bar{x}_{j}-x_{i} x_{j}\right) q_{i j} \\
& f\left(x^{\prime}\right)-f(x)=\sum_{i \in S}\left(\bar{x}_{i}-x_{i}\right) \Delta_{i}(x)-\sum_{j \in S} \sum_{j \notin S \backslash\{i\}}\left(\bar{x}_{i}-x_{i}\right) x_{i} q_{i j}+\sum_{i j \in S}\left(\bar{x}_{i} \bar{x}_{j}-\right. \\
& \left.x_{i} x_{j}\right) q_{i j}
\end{aligned}
$$


For each pair of elements $i, j$ in the second and the third terms we have $\bar{x}_{i} \bar{x}_{j}+x_{i} x_{j}-\bar{x}_{i} x_{j}-x_{i} \bar{x}_{j}=\left(\bar{x}_{i}-x_{i}\right)\left(\bar{x}_{j}-x_{j}\right)$

From this we get

$f\left(x^{\prime}\right)-f(x)=\sum_{i \in S}\left(\bar{x}_{i}-x_{i}\right) \Delta_{i}(x)+\sum_{\forall i, j \in S}\left(\bar{x}_{i}-x_{i}\right)\left(\bar{x}_{j}-x_{j}\right) \Delta_{i j}(x)$

which proves the first part of the theorem.

Note that we can substitute $\Delta_{i j}(x)$ for $q_{i j}$. From this the desired result follows immediately. We propose several methods to solve the transformed pseudoboolean problem.

Filtration and Sequential Fan(F\&F) (see Glover 13]) is a search process utilizing compound move strategies that have proven to be effective for a variety of combinatorial problems. Here we employ a simple version of F\&F utilizing a combination of $r$-flip moves. In the paragraph below we give a brief overview of our implementation.

F\&F organizes an aggressive search process utilizing a variety of different $r$-flip moves. The version we tested here starts with a set of locally optimal solutions found using 1-flip search. Then, for each solution in this set a second round of 1-flip moves is executed. If an improving move is found we accept that move and initiate a complete new round of 1 -flip moves. If an improving second flip move can not be found, we initiate a series of 3-flip moves starting with the best local optimal solutions found so far. This continues until a pre-set stopping criterion is satisfied or until no further improvement is realized.

\section{Computational Results}

Table 2 presents all subset of 2 alternatives and associated weights of each criterion, and payoff value of the selected subset of alternatives. In that, obviously subset $\left(a^{4}, a^{5}\right)$ is not any more the optimal solution as we have $v\left(a^{4}, a^{5}\right)=$ 1.391. However, the subset $\left(a^{1}, a^{2}\right)$ has the largest payoff of $v\left(a^{1}, a^{2}\right)=1.526$ which is optimal. Table 3 illustrates calculation of weights for each criterion for all subsets of 4 alternatives solved via Method 4. The dynamic nature of weights is clear from these tables. Obviously, when the interdependencies among

Table 2. Calculation of payoff when interdependencies are considered

\begin{tabular}{|c|c|c|c|c|}
\hline 2 alternatives $\left(a^{\imath}, a^{j}\right)$ & $w_{1}$ & $w_{2}$ & $w_{3}$ & $v\left(a^{\imath}, a^{j}\right)$ \\
\hline 1,2 & 0.23 & $0.39(1+0.30)=0.507$ & 0.38 & 1.526 \\
\hline 1,3 & 0.23 & 0.39 & 0.38 & 1.356 \\
\hline 1,4 & 0.23 & 0.39 & 0.38 & 1.379 \\
\hline 1,5 & 0.23 & 0.39 & 0.38 & 1.388 \\
\hline 2,3 & 0.23 & 0.39 & 0.38 & 1.420 \\
\hline 2,4 & 0.23 & 0.39 & 0.38 & 1.442 \\
\hline 2,5 & 0.23 & 0.39 & 0.38 & 1.452 \\
\hline 3,4 & 0.23 & 0.39 & 0.38 & 1.448 \\
\hline 3,5 & 0.23 & 0.39 & 0.38 & 1.457 \\
\hline 4,5 & 0.23 & $0.39(1+0.10)=0.429$ & $0.38(1-0.30)=0.266$ & 1.391 \\
\hline
\end{tabular}


Table 3. Calculation of weights for subset of 4 alternatives when interdependencies are considered

\begin{tabular}{|c|c|c|c|c|c|}
\hline & $a^{1}$ & $a^{2}$ & $a^{3}$ & $a^{4}$ & $a^{5}$ \\
\hline$w_{1}$ & 0.23 & 0.23 & 0.23 & 0.23 & \\
\hline$w_{2}$ & $0.39(1+0.30)=0.507$ & $0.39(1+0.30)=0.507$ & 0.39 & 0.39 & \\
\hline$w_{3}$ & 0.38 & 0.38 & 0.38 & 0.38 & \\
\hline$w_{1}$ & 0.23 & 0.23 & 0.23 & & 0.23 \\
\hline$w_{2}$ & $0.39(1+0.30)=0.507$ & $0.39(1+0.30)=0.507$ & 0.39 & & 0.39 \\
\hline$w_{3}$ & 0.38 & 0.38 & 0.38 & & 0.23 \\
\hline$w_{1}$ & 0.23 & 0.23 & & 0.23 & $0.10)=0.429$ \\
\hline$w_{2}$ & $0.39(1+0.30)=0.507$ & $0.39(1+0.30)=0.507$ & & $0.39(1+0.10)=0.429$ & $0.39(1+0.10)=0.266$ \\
\hline$w_{3}$ & 0.38 & 0.38 & & $0.38(1-0.30)=0.266$ & $0.38(1-0.30)=0.23$ \\
\hline$w_{1}$ & 0.23 & & 0.23 & 0.23 & 0.39 \\
\hline$w_{2}$ & 0.39 & & 0.39 & $0.39(1+0.10)=0.429$ & $0.39(1+0.10)=0.429$ \\
\hline$w_{3}$ & 0.38 & & 0.38 & $0.38(1-0.30)=0.266$ & $0.38(1-0.30)=0.266$ \\
\hline$w_{1}$ & & 0.23 & 0.23 & 0.23 & 0.23 \\
\hline$w_{2}$ & & 0.39 & 0.39 & $0.39(1+0.10)=0.429$ & $0.39(1+0.10)=0.429$ \\
\hline$w_{3}$ & & 0.38 & 0.38 & $0.38(1-0.30)=0.266$ & $0.38(1-0.30)=0.266$ \\
\hline
\end{tabular}

alternatives on different criteria are high the level of dynamism for each weight is also high. While considering the interdependencies among alternatives represents the practical nature of the problem, however calculating the dynamic weights is very difficult. If we need to select $m$ alternatives with highest total payoff we have to consider calculation of weights in all possible $m$ set of alternatives. And, in case, the problem is to select the best subset of alternatives (with no pre-specified number of elements) we have to calculate weights for all subset of alternatives. In both cases, the number of subsets is exponential.

\section{Summary and Conclusion}

In this paper we reported on modeling complex system problems with the pseudo-boolean formulation and proposed several solution approaches. The complex systems with very high-dimensional spaces often associate with more complicated variables. The pseudo-boolean formulation can reduce the variable dimension without loss important information. The preliminary results illustrate the attractiveness of this modeling method in term of generating the best alternative. In our continuing work, we intend to carry out further testing of pseudo-boolean formulation on cubic, quartic, even quintic variables.

\section{References}

1. Carpenter, G.A., Grossberg, S. and Rosen, D.B.: Fuzzy ART: Fast stable learning and categorization of analog patterns by an adaptive resonance system, Neural Networks 4 (1991) 759-771

2. Axelrod, R.: The Complexity of Cooperation: agent-based models of competition and collaboration. Princeton: Princeton University Press (1997) 
3. Döner D.: Heuristics and Cognition in Complex Systems, in: Methods of Heuristics, Groner R., Groner M. Bischof W.F. ed., Erlbaum, Hillsdale NJ(1983), 89-107

4. Holland J.H.: Studies of the spontaneous emergence of self-replicating systems using cellular automata and formal grammars. In A. Lindenmayer and G. Rozenberg, editors, Automata, Languages, Development, North-Holland, New York, (1976) 385-404

5. Stuart A.K.:The origins of order: self-organization and selection in evolution. Oxford University Press, Oxford, (1993)

6. Kaplan, D., Glass, L.: Understanding Nonlinear Dynamics, Springer-Verlag, NY (1995)

7. Ritchey, T.: Problem Structuring using Computer-Aided Morphological Analysis, Journal of the Operational Research Society, 57(2006), 792-801

8. Hayles, N. K. : Introduction: Complex Dynamics in Literature and Science, in Chaos and Order: Complex Dynamics in Literature and Science, (N. K. Hayles, ed.), Univ. Chicago Press, Chicago (1991) 1-36

9. Hammer, P., Rudeanu, S.: Boolean Methods in Operations Research and Related Areas, Springer, Berlin, Heidelberg, New York, (1968)

10. Boros, E., Hammer, P.: Pseudo-Boolean Optimization, Discrete Applied Mathematics, 123(2002), 155-225

11. Crama, Y., Hammer, P.: Boolean Functions: Theory, Algorithms and Applications (to be published in 2006)

12. Rajabi, S., Hipel,K.W., Kilgour, D.M.: Multiple criteria decision making under interdependence of actions - Systems, Man and Cybernetics, IEEE International Conference on Systems, Man and Cybernetics -Intelligent Systems for the 21st Century (1995)3, 2365-2370

13. Glover, F.: A Template for Scatter Search and Path Relinking, Working Paper, School of Business Administration, The University of Colorado. (1998)

14. Glover, F., Kochenberger, G., Alidaee, B.: Adaptive Memory Search for Binary Quadratic Programs, Management Science, 44(1998), 336-345 